\title{
JOINTLY MAXIMAL PRODUCTS IN WEIGHTED GROWTH SPACES
}

\author{
Janne Gröhn, José Ángel Peláez and Jouni Rättyä \\ University of Eastern Finland, Department of Physics and Mathematics \\ P.O. Box 111, 80101 Joensuu, Finland; janne.grohn@uef.fi \\ Universidad de Málaga, Departamento de Análisis Matemático \\ Campus de Teatinos, 29071 Málaga, Spain; japelaez@uma.es \\ University of Eastern Finland, Department of Physics and Mathematics \\ P.O. Box 111, 80101 Joensuu, Finland; jouni.rattya@uef.fi
}

\begin{abstract}
It is shown that for any positive, non-decreasing, continuous and unbounded doubling function $\omega$ on $[0,1)$, there exist two analytic infinite products $f_{0}$ and $f_{1}$ such that the asymptotic relation $\left|f_{0}(z)\right|+\left|f_{1}(z)\right| \asymp \omega(|z|)$ is satisfied for all $z$ in the unit disc. It is also shown that both functions $f_{j}$ for $j=0,1$ satisfy $T\left(r, f_{j}\right) \asymp \log \omega(r)$, as $r \rightarrow 1^{-}$, and hence give examples of analytic functions for which the Nevanlinna characteristic admits the regular slow growth induced by $\omega$.
\end{abstract}

\section{Introduction and results}

Let $\mathcal{H}(\mathbf{D})$ denote the algebra of all analytic functions in the unit disc $\mathbf{D}$ of the complex plane C. To consider the growth and the zero distribution of functions in $\mathcal{H}(\mathbf{D})$, we use the following classical notation. The non-integrated counting function $n(r, f, 0)$ counts the zeros of $f$ in $\{z \in \mathbf{C}:|z| \leq r\}$ according to multiplicities. Quantities $M_{\infty}(r, f), M_{p}(r, f)$, where $0<p<\infty, N(r, f, a)$, where $a \in \mathbf{C}$, and $T(r, f)$ denote the maximum modulus of $f$, the $L^{p}$-mean of $f$, the integrated counting function of a-points of $f$ and the Nevanlinna characteristic of $f$, respectively. We also employ the notation $a \asymp b$, which is equivalent to the conditions $a \lesssim b$ and $b \lesssim a$, where the former means that there exists a constant $C>0$ such that $a \leq C b$, and the latter is defined analogously.

Let $\omega:[0,1) \rightarrow(0, \infty)$ be non-decreasing, continuous and unbounded. Such a function $\omega$ is said to be doubling, if there exists a constant $B>1$ such that

$$
\omega(1-r / 2) \leq B \omega(1-r), \quad 0<r \leq 1 .
$$

The following result shows that for any doubling function $\omega$ there exist two jointly maximal products in the sense that the sum of their moduli behaves asymptotically as $\omega(|z|)$ in $\mathbf{D}$.

doi:10.5186/aasfm.2014.3901

2010 Mathematics Subject Classification: Primary 30J99.

Key words: Doubling function, infinite product, zero distribution.

This research was supported in part by the Ramón y Cajal program of MICINN (Spain), Ministerio de Educación y Ciencia, Spain, (MTM2011-25502), from La Junta de Andalucía, (FQM210) and (P09-FQM-4468), MICINN- Spain ref. MTM2011-26538, and the Academy of Finland \#258125. 
Theorem 1. Let $\omega:[0,1) \rightarrow(0, \infty)$ be doubling. Then, there exist $f_{0}, f_{1} \in$ $\mathcal{H}(\mathbf{D})$ such that

$$
\left|f_{0}(z)\right|+\left|f_{1}(z)\right| \asymp \omega(|z|), \quad z \in \mathbf{D}
$$

where both functions $f_{j}$ for $j=0,1$ satisfy $n\left(r, f_{j}, 0\right)=\mathrm{O}\left((1-r)^{-1}\right)$, as $r \rightarrow 1^{-}$. Moreover,

$$
M_{p}\left(r, f_{j}\right) \asymp \omega(r), \quad r \rightarrow 1^{-},
$$

for all $0<p \leq \infty$, and

$$
T\left(r, f_{j}\right) \asymp N\left(r, f_{j}, a\right) \asymp \log \omega(r), \quad r \rightarrow 1^{-},
$$

for all $a \in \mathbf{C}$.

The main advantage of our self-contained and constructive proof of Theorem 1 compared to the existing literature is that the zero distribution of the products $f_{0}$ and $f_{1}$ is explicit. These products are similar to those applied to study the zero distribution of functions in weighted Bergman spaces [7, Section 3]. Note also that our argument gives an alternative way to prove [7, Theorem 3.15], whose original proof is based on certain lacunary series. In fact, Theorem 1 generalizes [7, Theorem 3.15] to doubling functions.

The existence of $f_{0}, f_{1} \in \mathcal{H}(\mathbf{D})$ such that the asymptotic relation (2) is satisfied for a given doubling function $\omega$ was recently proved in [1, Lemma 1]. To see that this result is equivalent to [6, Theorem 1.1], that was published essentially at the same time as [1], we may argue as follows. If $\omega$ is doubling, then $\psi(x)=\omega(1-1 / x)$ is almost subnormal; see [6] for the definitions. Conversely, if $\psi$ is almost subnormal, then by means of $[6$, Lemma 2.1] there exists a function $\phi \asymp \psi$ such that $\omega(r)=\phi(1 /(1-r))$ is doubling. Proofs of [1, Lemma 1] and [6, Theorem 1.1] rest upon the use of lacunary series, which have been the key tool to solve similar problems in the existing literature. The pioneering result [8, Proposition 5.4], which concerns (2) for $\omega(r)=1 /(1-r)$, have been a source of inspiration for several authors. For example, [4, Theorem 1.2] proves (2) for $\omega(r)=-\log (1-r)$. It is well known that (2) has many applications in the operator theory; for details, we refer to $[1,6]$.

Construction of a product whose Nevanlinna characteristic admits a pregiven asymptotic growth has been studied by several authors. In particular, it is known that whenever $\Lambda(r)$ exceeds the growth of $-\log (1-r)$ as $r \rightarrow 1^{-}$, then there exists a product $f$ whose Nevanlinna characteristic behaves asymptotically as $\Lambda(r)[9$, Theorem 1]. The asymptotic formula (4) shows that we can find an infinite analytic product in $\mathbf{D}$ such that its Nevanlinna characteristic grows asymptotically as the logarithm of a pregiven doubling $\omega$, and hence we can prescribe characteristics growing slower than $-\log (1-r)$ as $r \rightarrow 1^{-}$. The method and the construction in the proof of Theorem 1 are different from those employed in [9].

The current state of the development concerning complex linear differential equation $f^{\prime \prime}+A(z) f=0$ in $\mathbf{D}$ allows us to deduce a significant amount of information on solutions $f$, whenever we can analyze the coefficient $A$ in detail. If we take $A$ to be one of the functions $f_{0}$ and $f_{1}$ in the Theorem 1 , then we get an important and intriguing family of examples of such differential equations. These particular equations are way too complicated to be solved explicitly, but the growth and the oscillation of their solutions are well understood due to the asymptotic properties satisfied by the coefficient $A$. To be brief with regards to this matter, we settle to mention two cases 
in the recent literature of which the first one concerns polynomial regular functions. This class of regularly growing analytic functions in $\mathbf{D}$ arises naturally in the theory of ODEs [3]. In the sense of linear differential equations, polynomial regular functions play a similar role in the unit disc as polynomials do in the complex plane. For a more general example, see [2].

\section{Proof of Theorem 1}

The proof of Theorem 1 is divided in several steps. The point of departure is the construction of the infinite products $f_{0}, f_{1} \in \mathcal{H}(\mathbf{D})$, which is followed by a discussion of their growth. Finally, we consider the asserted asymptotic properties of the products $f_{0}$ and $f_{1}$.

2.1. Construction of the products. Before going into the details of the construction, we note the following lemmas on doubling functions.

Lemma 2. Let $\omega:[0,1) \rightarrow(0, \infty)$ be doubling. If $B>1$ is the constant in (1), then

$$
\omega(t) \leq C\left(\frac{1-r}{1-t}\right)^{\alpha} \omega(r), \quad 0 \leq r \leq t<1
$$

where $C=\max \left\{B \omega(1 / 2) / \omega(0), B^{2}\right\}$ and $\alpha=\log _{2} B$.

Conversely, it is obvious that, if $\omega:[0,1) \rightarrow(0, \infty)$ is non-decreasing, continuous, unbounded and it satisfies (5) for some $C>1$ and $\alpha>0$, then $\omega$ must be doubling.

Proof of Lemma 2. Since $\omega$ is doubling, (1) implies $\omega(t) \leq B \omega(2 t-1)$ for all $t \in\left[2^{-1}, 1\right)$. Moreover, if $0 \leq r \leq t<1$, then there exist unique constants $j, k \in \mathbf{N} \cup\{0\}, j \geq k$, such that $t \in\left[1-2^{-j}, 1-2^{-j-1}\right)$ and $r \in\left[1-2^{-k}, 1-2^{-k-1}\right)$. If $k=0$, then

$$
\omega(t) \leq B^{j} \omega\left(2^{j}(t-1)+1\right) \leq \frac{B^{j} \omega(1 / 2)}{\omega(0)} \omega(r) \leq \frac{B \omega(1 / 2)}{\omega(0)}\left(\frac{1-r}{1-t}\right)^{\log _{2} B} \omega(r),
$$

while if $k>0$, then

$$
\omega(t) \leq B^{j-k+1} \omega\left(2^{j-k+1}(t-1)+1\right) \leq B^{j-k+1} \omega(r) \leq B^{2}\left(\frac{1-r}{1-t}\right)^{\log _{2} B} \omega(r) .
$$

The assertion follows.

The second lemma introduces a sequence of natural numbers depending on the growth of the doubling function $\omega$. This sequence is the foundation of our construction.

Lemma 3. Let $\omega:[0,1) \rightarrow(0, \infty)$ be doubling. Then, there exist a sequence $\left\{n_{k}\right\}_{k=1}^{\infty}$ of natural numbers, real constants $\lambda$ and $\mu$, and a constant $d \in(0,1)$ such that the sequence $\left\{a_{k}\right\}_{k=1}^{\infty}$, defined by

$$
a_{k}=\frac{\omega\left(1-1 / n_{k+2}\right)}{\omega\left(1-1 / n_{k}\right)}, \quad k \in \mathbf{N},
$$

satisfies

$$
1<\lambda \leq a_{k} \leq \mu<\infty, \quad \frac{\log a_{k+1}}{\log a_{k}}<d \frac{n_{k+1}}{n_{k}}, \quad k \in \mathbf{N}
$$


Proof. Let $\alpha>0$ and $C>1$ be the constants ensured by Lemma 2. Now, let $\gamma$ be a sufficiently large real constant such that

$$
2^{\gamma-\alpha} C^{-1}>1, \quad \frac{2 \gamma+\alpha+\log _{2} C}{2 \gamma-\alpha-\log _{2} C}<\frac{1}{2^{1 / \alpha}}\left(\frac{2^{\gamma / \alpha}}{C^{1 / \alpha}}-1\right) .
$$

Take $t_{1}=1 / 2$, and define the sequence $\left\{t_{k}\right\}_{k=1}^{\infty}$ inductively by $\omega\left(t_{k+1}\right) / \omega\left(t_{k}\right)=2^{\gamma}$ for $k \in \mathbf{N}$. Let $n_{k}=$ floor $\left(\left(1-t_{k}\right)^{-1}\right)$, where floor $(x)=\max \{n \in \mathbf{N}: n \leq x\}$. By means of Lemma 2, and the estimates $2^{-1} \leq n_{k}\left(1-t_{k}\right) \leq 1$, we may define $1<\lambda<\mu<\infty$ by

$$
a_{k} \leq \frac{C \omega\left(t_{k+2}\right)}{\left(n_{k}\left(1-t_{k}\right)\right)^{\alpha} \omega\left(t_{k}\right)} \leq 2^{\alpha} C \frac{\omega\left(t_{k+2}\right)}{\omega\left(t_{k+1}\right)} \frac{\omega\left(t_{k+1}\right)}{\omega\left(t_{k}\right)}=2^{2 \gamma+\alpha} C=\mu,
$$

and

$$
a_{k} \geq \frac{\left(n_{k+2}\left(1-t_{k+2}\right)\right)^{\alpha} \omega\left(t_{k+2}\right)}{C \omega\left(t_{k}\right)} \geq \frac{1}{2^{\alpha} C} \frac{\omega\left(t_{k+2}\right)}{\omega\left(t_{k+1}\right)} \frac{\omega\left(t_{k+1}\right)}{\omega\left(t_{k}\right)}=2^{2 \gamma-\alpha} C^{-1}=\lambda,
$$

since these inequalities hold for all $k \in \mathbf{N}$. By Lemma 2 we conclude

$$
\begin{aligned}
\frac{n_{k+1}}{n_{k}} & >\left(\frac{1}{1-t_{k+1}}-1\right)\left(1-t_{k}\right)>\frac{1-t_{k}}{1-t_{k+1}}-1 \\
& \geq \frac{1}{C^{1 / \alpha}}\left(\frac{\omega\left(t_{k+1}\right)}{\omega\left(t_{k}\right)}\right)^{1 / \alpha}-1=\frac{2^{\gamma / \alpha}}{C^{1 / \alpha}}-1, \quad k \in \mathbf{N},
\end{aligned}
$$

and further by (7), we have

$$
\frac{\log a_{k+1}}{\log a_{k}} \leq \frac{\log \mu}{\log \lambda}=\frac{2 \gamma+\alpha+\log _{2} C}{2 \gamma-\alpha-\log _{2} C}<\frac{1}{2^{1 / \alpha}}\left(\frac{2^{\gamma / \alpha}}{C^{1 / \alpha}}-1\right)<\frac{1}{2^{1 / \alpha}} \frac{n_{k+1}}{n_{k}}
$$

This confirms the last inequality in (6) for $d=2^{-1 / \alpha}$.

Let $\left\{n_{k}\right\}_{k=1}^{\infty}$ be the sequence ensured by Lemma 3, and define

$$
f_{j}(z)=\prod_{k=1}^{\infty} \frac{1+a_{2 k+j} z^{n_{2 k+j}}}{1+a_{2 k+j}^{-1} z^{n_{2 k+j}}}, \quad z \in \mathbf{D}, \quad j=0,1 .
$$

Evidently both functions $f_{j}$ belong to $\mathcal{H}(\mathbf{D})$, since all factors are bounded functions in $\mathbf{D}$, and according to (6) the sum

$$
\sum_{k=1}^{\infty}\left|\frac{1+a_{2 k+j} z^{n_{2 k+j}}}{1+a_{2 k+j}^{-1} z^{n_{2 k+j}}}-1\right| \leq \sum_{k=1}^{\infty} \frac{a_{2 k+j}-a_{2 k+j}^{-1}}{1-a_{2 k+j}^{-1}}|z|^{n_{2 k+j}} \leq(1+\mu) \sum_{k=1}^{\infty}|z|^{n_{2 k+j}}
$$

converges uniformly on compact subsets of $\mathbf{D}$.

2.2. Growth estimates for the maximum modulus of the products. To estimate the growth of $f_{j}$ for $j=0,1$ we define $r_{2 m+j}=e^{-1 / n_{2 m+j}}$ for $m \in \mathbf{N}$, and write

$$
\left|f_{j}(z)\right|=\left|\prod_{k=1}^{m} a_{2 k+j} \frac{a_{2 k+j}^{-1}+z^{n_{2 k+j}}}{1+a_{2 k+j}^{-1} z^{n_{2 k+j}}}\right|\left|\prod_{k=1}^{\infty} \frac{1+a_{2(m+k)+j} z^{n_{2(m+k)+j}}}{1+a_{2(m+k)+j}^{-1} z^{n_{2(m+k)+j}}}\right| .
$$

First, we prove that the infinite subproduct in (9) is bounded in $\mathbf{D}$. To this end, let $\tau=2^{\gamma / \alpha} C^{-1 / \alpha}-1$ be the lower bound in (8). According to (7) we know that $\tau>1$, 
and

$$
\frac{n_{2(m+k)+j}}{n_{2 m+j}}=\frac{n_{2(m+k)+j}}{n_{2(m+k)+j-1}} \cdots \frac{n_{2 m+j+1}}{n_{2 m+j}} \geq \tau^{2 k}, \quad k, m \in \mathbf{N} .
$$

Since $h_{1}(x)=(y+x) /(1+y x)$ is increasing on $[0,1)$ for each $y \in[0,1)$, we obtain

$$
\begin{aligned}
\left|\frac{1+a_{2(m+k)+j} z^{n_{2(m+k)+j}}}{1+a_{2(m+k)+j}^{-1} z^{n_{2(m+k)+j}}}\right| & =a_{2(m+k)+j}\left|\frac{a_{2(m+k)+j}^{-1}+z^{n_{2(m+k)+j}}}{1+a_{2(m+k)+j}^{-1} z^{n_{2(m+k)+j}}}\right| \\
& \leq a_{2(m+k)+j} \frac{a_{2(m+k)+j}^{-1}+|z|^{n_{2(m+k)+j}}}{1+a_{2(m+k)+j}^{-1}|z|^{n_{2(m+k)+j}}} \\
& <\frac{1+a_{2(m+k)+j}\left(\frac{1}{e}\right)^{\frac{n_{2(m+k)+j}}{n_{2 m+j}}}}{1+a_{2(m+k)+j}^{-1}\left(\frac{1}{e}\right)^{\frac{n_{2(m+k)+j}}{n_{2 m+j}}}}
\end{aligned}
$$

for $|z|<r_{2 m+j}$ and $k, m \in \mathbf{N}$. Moreover, since $h_{2}(x, y)=(1+x y) /\left(1+x^{-1} y\right)$ is increasing in both variables, provided that $x>1$ and $0 \leq y<1$, estimates (6), (10) and (11) imply

$$
\left|\prod_{k=1}^{\infty} \frac{1+a_{2(m+k)+j} z^{n_{2(m+k)+j}}}{1+a_{2(m+k)+j}^{-1} z^{n_{2(m+k)+j}}}\right|<\prod_{k=1}^{\infty} \frac{1+\mu\left(\frac{1}{e}\right)^{\tau^{2 k}}}{1+\mu^{-1}\left(\frac{1}{e}\right)^{\tau^{2 k}}} \leq C^{\star}<\infty,
$$

for $|z|<r_{2 m+j}$ and $m \in \mathbf{N}$, where $C^{\star}>0$ is a constant independent of $m \in \mathbf{N}$. Second, we proceed to derive an upper estimate for the maximum modulus of $f_{j}$. By means of (6), (9), (12) and the inequality $1-x \leq e^{-x}$ for $x \geq 0$, we get

$$
\begin{aligned}
\left|f_{j}(z)\right| & <C^{\star} \prod_{k=1}^{m} a_{2 k+j}=C^{\star} \frac{\omega\left(1-1 / n_{2(m+1)+j}\right)}{\omega\left(1-1 / n_{2+j}\right)} \leq C^{\star} \mu \frac{\omega\left(1-1 / n_{2 m+j}\right)}{\omega\left(1-1 / n_{2+j}\right)} \\
& \leq C^{\star} \mu \frac{\omega\left(r_{2 m+j}\right)}{\omega\left(1-1 / n_{2+j}\right)}, \quad|z|<r_{2 m+j}, \quad m \in \mathbf{N} .
\end{aligned}
$$

If $|z| \geq r_{2+j}$, then $r_{2(m-1)+j} \leq|z|<r_{2 m+j}$ for some $m \in \mathbf{N} \backslash\{1\}$. Note that by (10) there exists $t \in \mathbf{N}$ such that $n_{2(m+t)+j}>2 n_{2 m+j}$ for all $m \in \mathbf{N}$. Since $e^{-x} \leq 1-x / 2$ for $0 \leq x \leq 1$, we conclude

$$
r_{2 m+j} \leq 1-\left(2 n_{2 m+j}\right)^{-1}<1-1 / n_{2(m+t)+j}, \quad m \in \mathbf{N} .
$$

Then (6), (13) and the inequality $1-x \leq e^{-x}$ for $0 \leq x \leq 1$, give

$$
\begin{aligned}
\left|f_{j}(z)\right| & <C^{\star} \mu \frac{\omega\left(1-1 / n_{2(m+t)+j}\right)}{\omega\left(1-1 / n_{2+j}\right)} \leq C^{\star} \mu^{2+t} \frac{\omega\left(1-1 / n_{2(m-1)+j}\right)}{\omega\left(1-1 / n_{2+j}\right)} \\
& \leq C^{\star} \mu^{2+t} \frac{\omega\left(r_{2(m-1)+j}\right)}{\omega\left(1-1 / n_{2+j}\right)} \leq C^{\star} \mu^{2+t} \frac{\omega(|z|)}{\omega\left(1-1 / n_{2+j}\right)} .
\end{aligned}
$$

Consequently, the maximum modulus of $f_{j}$ satisfies

$$
M_{\infty}\left(r, f_{j}\right)=\max _{|z|=r}\left|f_{j}(z)\right| \lesssim \omega(r), \quad 0 \leq r<1 .
$$

2.3. Growth estimates for the minimum modulus of the products. The following discussion shows that the difference between the maximum modulus and 
the minimum modulus of $f_{j}$ for $j=0,1$ is small in a large subset of the unit disc. Define $E_{j}=\bigcup_{m=1}^{\infty} I_{2 m+j}$, where $I_{2 m+j}$ is the closed interval whose endpoints are

$$
\min I_{2 m+j}=\left(a_{2 m+j}^{-n_{2 m+j}^{-1}}\right)^{1-\delta}\left(a_{2(m+1)+j}^{-n_{2(m+1)+j}^{-1}}\right)^{\delta}, \quad m \in \mathbf{N},
$$

and

$$
\max I_{2 m+j}=\left(a_{2 m+j}^{-n_{2 m+j}^{-1}}\right)^{\delta \frac{n_{2 m+j}}{n_{2 m+1+j}}}\left(a_{2(m+1)+j}^{-n_{2(m+1)+j}^{-1}}\right)^{1-\delta \frac{n_{2 m+j}}{n_{2 m+1+j}}}, \quad m \in \mathbf{N} .
$$

Here $0<\delta<1$ is a sufficiently small constant, which is to be determined later. According to (6) all elements in the sequence $\left\{a_{m}^{-1 / n_{m}}\right\}_{m=1}^{\infty}$ belong to the interval $(0,1)$, this sequence is strictly increasing, and it converges to 1 , as $m \rightarrow \infty$. Moreover, $I_{2 m+j} \subset\left(a_{2 m+j}^{-1 / n_{2 m+j}}, a_{2(m+1)+j}^{-1 / n_{2(m+1)+j}}\right)$ for all $m \in \mathbf{N}$. First, we prove that the infinite subproduct in (9) is uniformly bounded away from zero for $|z| \in E_{j}$. If $|z| \in I_{2 m+j}$, then $|z|^{n_{2(m+k)+j}}<a_{2(m+k)+j}^{-1}$ for all $k \in \mathbf{N}$, and therefore

$$
\begin{aligned}
\left|\frac{1+a_{2(m+k)+j} z^{n_{2(m+k)+j}}}{1+a_{2(m+k)+j}^{-1} z^{n_{2(m+k)+j}}}\right| & \geq a_{2(m+k)+j} \frac{a_{2(m+k)+j}^{-1}-|z|^{n_{2(m+k)+j}}}{1-a_{2(m+k)+j}^{-1}|z|^{n_{2(m+k)+j}}} \\
& =\frac{1-a_{2(m+k)+j}|z|^{n_{2(m+k)+j}}}{1-a_{2(m+k)+j}^{-1}|z|^{n_{2(m+k)+j}}}
\end{aligned}
$$

for $|z| \in I_{2 m+j}$ and $k, m \in \mathbf{N}$. Since $h_{3}(x, y)=(1-x y) /\left(1-x^{-1} y\right)$ is decreasing in both variables, when $x>1$ and $0 \leq y<1$, estimates (6), (10) and (15) imply that there exists a constant $C^{*}>0$, independent of $m \in \mathbf{N}$, such that

$$
\begin{aligned}
& \left|\prod_{k=1}^{\infty} \frac{1+a_{2(m+k)+j} z^{n_{2(m+k)+j}}}{1+a_{2(m+k)+j}^{-1} z^{n_{2(m+k)+j}}}\right| \\
& \geq \prod_{k=1}^{\infty} \frac{1-a_{2(m+k)+j}\left(a_{2 m+j}^{-\delta} a_{2(m+1)+j}^{-\frac{n_{2 m+1+j}}{n_{2(m+1)+j}}\left(1-\delta \frac{n_{2 m+j}}{n_{2 m+1+j}}\right)}\right)^{\frac{n_{2(m+k)+j}}{n_{2 m+1+j}}}}{1-a_{2(m+k)+j}^{-1}\left(a_{2 m+j}^{-\delta} a_{2(m+1)+j}^{-\frac{n_{2 m+1+j}}{n_{2(m+1)+j}}\left(1-\delta \frac{n_{2 m+j}}{n_{2 m+1+j}}\right)}\right)^{\frac{n_{2(m+k)+j}}{n_{2 m+1+j}}}} \\
& \geq \prod_{k=1}^{\infty} \frac{1-\mu\left(\lambda^{-\delta}\right)^{\tau^{2 k-1}}}{1-\mu^{-1}\left(\lambda^{-\delta}\right)^{\tau^{2 k-1}}} \geq C^{*}, \quad|z| \in I_{2 m+j}, \quad m \in \mathbf{N} .
\end{aligned}
$$

Second, we proceed to estimate the minimum modulus of $f_{j}$ on $E_{j}$. Note that the last inequality in (6) implies

$$
\begin{aligned}
a_{2 k+j}|z|^{n_{2 k+j}} & \geq a_{2 k+j}\left(a_{2 m+j}^{-\frac{1}{n_{2 m+j}}(1-\delta)} a_{2(m+1)+j}^{-\frac{1}{n_{2(m+1)+j}} \delta}\right)^{n_{2 k+j}} \\
& =\frac{a_{2 k+j}}{a_{2 m+j}^{\frac{n_{2 k+j}}{n_{2 m+j}}(1-\delta)} a_{2(m+1)+j}^{\frac{n_{2 k+j}}{n_{2(m+1)+j}}} \geq \frac{a_{2 k+j}}{a_{2 k+j}^{d^{2(m-k)}(1-\delta)} a_{2 k+j}^{d^{2(m+1-k)} \delta}}} \\
& \geq \frac{a_{2 k+j}}{a_{2 k+j}^{1-\delta} a_{2 k+j}^{d \delta}}=a_{2 k+j}^{\delta(1-d)} \geq \lambda^{\delta(1-d)}>1
\end{aligned}
$$


for $|z| \in I_{2 m+j}$ when $1 \leq k \leq m$, and $m \in \mathbf{N}$; in particular, $|z|^{n_{2 k+j}}>a_{2 k+j}^{-1}$. Moreover, choose $t \in \mathbf{N}$ sufficiently large such that $1-\lambda^{-1} \geq \tau^{-2 t}$. Since $h_{4}(x)=$ $1-(1-a) x^{-1}-a^{1 / x} \geq 0$ for all $x \in[1, \infty)$, provided that $a \in(0,1)$, by applying $(6)$ and (10), we obtain

$$
\begin{aligned}
|z| & \leq a_{2(m+1)+j}^{-1 / n_{2(m+1)+j}} \leq \lambda^{-1 / n_{2(m+1)+j}} \leq 1-\left(1-\lambda^{-1}\right) n_{2(m+1)+j}^{-1} \\
& \leq 1-\tau^{-2 t} n_{2(m+1)+j}^{-1} \leq 1-1 / n_{2(m+1+t)+j}, \quad|z| \in I_{2 m+j}, \quad m \in \mathbf{N} .
\end{aligned}
$$

Therefore (9), (16) and (18) yield

$$
\begin{aligned}
\left|f_{j}(z)\right| & \geq C^{*} \prod_{k=1}^{m} a_{2 k+j}\left|\frac{a_{2 k+j}^{-1}+z^{n_{2 k+j}}}{1+a_{2 k+j}^{-1} z^{n_{2 k+j}}}\right| \\
& =C^{*} \frac{\omega\left(1-1 / n_{2(m+1)+j}\right)}{\omega\left(1-1 / n_{2+j}\right)} \prod_{k=1}^{m}\left|\frac{a_{2 k+j}^{-1}+z^{n_{2 k+j}}}{1+a_{2 k+j}^{-1} z^{n_{2 k+j}}}\right| \\
& \geq C^{*} \frac{\omega\left(1-1 / n_{2(m+1+t)+j}\right)}{\mu^{t} \omega\left(1-1 / n_{2+j}\right)} \prod_{k=1}^{m} \frac{|z|^{n_{2 k+j}}-a_{2 k+j}^{-1}}{1-a_{2 k+j}^{-1}|z|^{n_{2 k+j}}} \\
& \geq C^{*} \frac{\omega(|z|)}{\mu^{t} \omega\left(1-1 / n_{2+j}\right)} \prod_{k=1}^{m} \frac{|z|^{n_{2 k+j}}-a_{2 k+j}^{-1}}{1-a_{2 k+j}^{-1}|z|^{n_{2 k+j}}}
\end{aligned}
$$

for $|z| \in I_{2 m+j}$ and $m \in \mathbf{N}$. For our purposes, it suffices to show that the product in the last line of (19) is uniformly bounded away from zero for $|z| \in E_{j}$. To simplify computations, we prove that the reciprocal of this product is uniformly bounded for such values of $z$. Now, since $\log x \leq x-1$ for $x \geq 1$, we have

$$
\begin{aligned}
\prod_{k=1}^{m} \frac{1-a_{2 k+j}^{-1}|z|^{n_{2 k+j}}}{|z|^{n_{2 k+j}}-a_{2 k+j}^{-1}} & =\exp \left(\sum_{k=1}^{m} \log \frac{1-a_{2 k+j}^{-1}|z|^{n_{2 k+j}}}{|z|^{n_{2 k+j}}-a_{2 k+j}^{-1}}\right) \\
& \leq \exp \left((1+\mu) \sum_{k=1}^{m} \frac{1-|z|^{n_{2 k+j}}}{a_{2 k+j}|z|^{n_{2 k+j}}-1}\right)
\end{aligned}
$$

for $|z| \in I_{2 m+j}$ and $m \in \mathbf{N}$. By means of (10), (17), and the estimate $1-e^{-x} \leq x$ for $x \geq 0$, we conclude

$$
\begin{aligned}
\prod_{k=1}^{m} \frac{1-a_{2 k+j}^{-1}|z|^{n_{2 k+j}}}{|z|^{n_{2 k+j}}-a_{2 k+j}^{-1}} & \leq \exp \left(\frac{1+\mu}{\lambda^{\delta(1-d)}-1} \sum_{k=1}^{m}\left(1-|z|^{n_{2 k+j}}\right)\right) \\
& \leq \exp \left(\frac{1+\mu}{\lambda^{\delta(1-d)}-1} \sum_{k=1}^{m}\left(1-a_{2 m+j}^{-n_{2 k+j} / n_{2 m+j}}\right)\right) \\
& \leq \exp \left(\frac{1+\mu}{\lambda^{\delta(1-d)}-1} \log \mu \sum_{k=1}^{m} \frac{n_{2 k+j}}{n_{2 m+j}}\right) \\
& \leq \exp \left(\frac{1+\mu}{\lambda^{\delta(1-d)}-1} \log \mu \sum_{k=0}^{\infty} \frac{1}{\tau^{k}}\right)
\end{aligned}
$$


for $|z| \in I_{2 m+j}$ and $m \in \mathbf{N}$, which gives the desired uniform lower bound for the product in the last line of (19). Hence, by (14) and (19), we get

$$
\left|f_{j}(z)\right| \asymp \omega(|z|), \quad|z| \in E_{j}=\bigcup_{m=1}^{\infty} I_{2 m+j} .
$$

2.4. The covering property of the sets where the products are maximal. It remains to prove that the sets $E_{0}$ and $E_{1}$ induce a covering of $\left[\min I_{2}, 1\right)$. Note that the closed intervals $\left\{I_{2 m}\right\}_{m=1}^{\infty}$ are pairwise disjoint, which is also true for $\left\{I_{2 m+1}\right\}_{m=1}^{\infty}$. Consequently, it is sufficient to show that

$$
\min I_{2 m+1} \leq \max I_{2 m}, \quad \min I_{2(m+1)} \leq \max I_{2 m+1}, \quad m \in \mathbf{N} .
$$

We proceed to prove the first inequality in (21). By the definition of $I_{2 m+j}$, the first inequality in (21) is equivalent to

$$
a_{2 m+1}^{-\frac{1-\delta}{n_{2 m+1}}} a_{2 m+3}^{-\frac{\delta}{n_{2 m+3}}} \leq a_{2 m}^{-\frac{\delta}{n_{2 m+1}}} a_{2 m+2}^{-\frac{1}{n_{2 m+2}}\left(1-\delta \frac{n_{2 m}}{n_{2 m+1}}\right)}, \quad m \in \mathbf{N} .
$$

By taking the logarithm to the base $a_{2 m}$ on the both sides of (22), and then solving the resulting inequality with respect to $\delta$, we conclude that the first inequality in (22) is valid if and only if $\delta \leq T(m)$ for all $m \in \mathbf{N}$, where

$$
T(m)=\frac{\log _{a_{2 m}} a_{2 m+2}^{-\frac{1}{n_{2 m+2}}}-\log _{a_{2 m}} a_{2 m+1}^{-\frac{1}{n_{2 m+1}}}}{\frac{1}{n_{2 m+1}}-\log _{a_{2 m}} a_{2 m+1}^{-\frac{1}{n_{2 m+1}}}+\log _{a_{2 m}} a_{2 m+3}^{-\frac{1}{n_{2 m+3}}}+\log _{a_{2 m}} a_{2 m+2}^{-\frac{n_{2 m}}{n_{2 m+1} n_{2 m+2}}}} .
$$

Note that the denominator of $T(m)$ can be written in the form

$$
\begin{aligned}
& \log _{a_{2 m}} a_{2 m+3}^{-\frac{1}{n_{2 m+3}}}-\log _{a_{2 m}} a_{2 m+1}^{-\frac{1}{n_{2 m+1}}} \\
& \quad+\frac{n_{2 m}}{n_{2 m+1}}\left(\log _{a_{2 m}} a_{2 m+2}^{-\frac{1}{n_{2 m+2}}}-\log _{a_{2 m}} a_{2 m}^{-\frac{1}{n_{2 m}}}\right)>0, \quad m \in \mathbf{N},
\end{aligned}
$$

and hence $T(m)$ is strictly positive for all $m \in \mathbf{N}$. By means of (6) we get

$$
T(m) \geq \frac{(d-1) \log _{a_{2 m}} a_{2 m+1}^{-\frac{1}{n_{2 m+1}}}}{\frac{1}{n_{2 m+1}}-\log _{a_{2 m}} a_{2 m+1}^{-\frac{1}{n_{2 m+1}}}}=\frac{(1-d) \log _{a_{2 m}} a_{2 m+1}}{1+\log _{a_{2 m}} a_{2 m+1}}, \quad m \in \mathbf{N} .
$$

This implies that, if

$$
0<\delta \leq \frac{(1-d) \log _{\mu} \lambda}{1+\log _{\lambda} \mu}
$$

then the first inequality in (21) is satisfied for all $m \in \mathbf{N}$. The second inequality in (22) follows by a similar argument, and the choice (23) for $\delta$ is again adequate. We conclude that

$$
\left|f_{0}(z)\right|+\left|f_{1}(z)\right| \asymp \omega(|z|)
$$

for $|z| \geq \min I_{2}$. Finally, since $\left\{a_{m}^{-1 / n_{m}}\right\}_{m=1}^{\infty}$ is strictly increasing, (24) holds also for $|z| \leq \min I_{2}$, and hence $f_{0}$ and $f_{1}$ are analytic functions satisfying (2). 
2.5. Asymptotic properties of the products. Product $f_{j}$ for $j=0,1$ has exactly $n_{2 m+j}$ simple zeros on the each circle $\left\{z:|z|=s_{2 m+j}\right\}$, where $s_{2 m+j}=$ $a_{2 m+j}^{-1 / n_{2 m+j}}$ for $m \in \mathbf{N}$. Therefore, we obtain

$$
\begin{aligned}
n_{2 m+j} & \leq n\left(s_{2 m+j}, f_{j}, 0\right)=\sum_{k=1}^{m} n_{2 k+j}=n_{2 m+j} \sum_{k=1}^{m} \frac{1}{\frac{n_{2 m+j}}{n_{2 k+j}}} \\
& \leq n_{2 m+j} \sum_{k=0}^{\infty} \frac{1}{\tau^{k}} \lesssim n_{2 m+j}, \quad m \in \mathbf{N},
\end{aligned}
$$

by (10). By applying the estimates $1-x<\log x^{-1}<2(1-x)$, which are valid for $4^{-1}<x<1$, it follows that $n\left(s_{2 m+j}, f_{j}, 0\right) \asymp\left(1-s_{2 m+j}\right)^{-1}$ for all $m \in \mathbf{N}$. Consequently,

$$
n\left(r, f_{j}, 0\right)=\mathrm{O}\left((1-r)^{-1}\right), \quad r \rightarrow 1^{-} .
$$

Now we observe that $E_{0} \cup E_{1}=\left[\min I_{2}, 1\right)$, so it is not possible that $\underline{d}\left(E_{0}\right)=$ $\underline{d}\left(E_{1}\right)=0$, where

$$
\underline{d}(F)=\liminf _{r \rightarrow 1^{-}} \frac{m(F \cap[r, 1))}{1-r}
$$

is the lower density of the set $F \subset[0,1)$, and where $m$ denotes the Lebesgue measure. Consequently, for some $j=0,1$ we have $\underline{d}\left(E_{j}\right)>0$, which together with the nature of the sets $E_{j}$, implies that $\underline{d}\left(E_{j}\right)>0$ for both $j=0,1$. Consequently, (20) holds outside a set $E_{j}^{\star}=[0,1) \backslash E_{j}$, which satisfies

$$
\bar{d}\left(E_{j}^{\star}\right)=\limsup _{r \rightarrow 1^{-}} \frac{m\left(E_{j}^{\star} \cap[r, 1)\right)}{1-r}<1, \quad j=0,1 .
$$

So, (20), [5, Lemma 2] and Lemma 2 yield $M_{p}\left(r, f_{j}\right) \asymp \omega(r)$, as $r \rightarrow 1^{-}$, where the constants in the asymptotic relation are independent of $0<p \leq \infty$. This proves (3). On the other hand, for any $a \in \mathbf{C}$, Jensen's formula and (20) imply that $N\left(r, f_{j}, a\right) \asymp$ $\log \omega(r)$ for $r \in[0,1) \backslash E_{0}^{\star}$. The fact that the same estimate holds also without the exceptional set $E_{0}^{\star}$ follows again from [5, Lemma 2] and Lemma 2. Furthermore, $\log \omega(r) \asymp N\left(r, f_{j}, 0\right) \lesssim T\left(r, f_{j}\right) \leq \log M_{\infty}\left(r, f_{j}\right) \asymp \log \omega(r)$, as $r \rightarrow 1^{-}$, again by Jensen's formula. This completes the proof of Theorem 1.

Acknowledgements. We thank the referee for a careful reading of the manuscript, and in particular, for pointing out the connection between [6, Theorem 1.1] and the asymptotic relation (2). The first author is also grateful to the Autonomous University of Barcelona for hospitality during the academic year 2012-2013.

\section{References}

[1] Abakumov, E., and E. Doubtsov: Reverse estimates in growth estimates. - Math. Z. 271, 2012, 399-413.

[2] Chuaqui, M., J. Gröhn, J. Heittokangas, and J. RÄTtyä: Zero separation results for solutions of second order linear differential equations. - Adv. Math. 245, 2013, 382-422.

[3] Fenton, P., J. Gröhn, J. Heittokangas, J. Rossi, and J. RÄttyä: On $\alpha$-polynomial regular functions, with applications to ODEs. - Proc. Edinb. Math. Soc. (to appear).

[4] Girela, D., J. A. Peláez, F. Pérez-González, and J. RÄttyä: Carleson measures for the Bloch space. - Integral Equations Operator Theory 61:4, 2008, 511-547. 
[5] Heittokangas, J.: A survey on Blaschke-oscillatory differential equations, with updates. Fields Inst. Commun. 65, 2013, 43-98.

[6] Kwon, E. G., and M. Pavlović: Bibloch mappings and composition operators from Bloch type spaces to BMOA. - J. Math. Anal. Appl. 382, 2011, 303-313.

[7] PelÁez, J.A., and J. RÄтtYä: Weighted Bergman spaces induced by rapidly increasing weights. - Mem. Amer. Math. Soc. 227:1066, 2014.

[8] Ramey, W., and D. Ullrich: Bounded mean oscillation of Bloch pull-backs. - Math. Ann. 291:4, 1991, 591-606.

[9] Shea, D. F.: Functions analytic in a finite disk and having asymptotically prescribed characteristic. - Pacific J. Math. 17, 1966, 549-560.

Received 24 December 2012 • Accepted 6 May 2013 